\title{
Synchronization of oscillators with long range interaction: Phase transition and anomalous finite size effects
}

\section{Marodi, M.; D'ovidio, Francesco; Vicsek, T.}

Published in:

Physical Review E. Statistical, Nonlinear, and Soft Matter Physics

Link to article, DOI:

10.1103/PhysRevE.66.011109

Publication date:

2002

Document Version

Publisher's PDF, also known as Version of record

Link back to DTU Orbit

Citation (APA):

Marodi, M., D'ovidio, F., \& Vicsek, T. (2002). Synchronization of oscillators with long range interaction: Phase transition and anomalous finite size effects. Physical Review E. Statistical, Nonlinear, and Soft Matter Physics, 66(1), 011109. https://doi.org/10.1103/PhysRevE.66.011109

\section{General rights}

Copyright and moral rights for the publications made accessible in the public portal are retained by the authors and/or other copyright owners and it is a condition of accessing publications that users recognise and abide by the legal requirements associated with these rights.

- Users may download and print one copy of any publication from the public portal for the purpose of private study or research.

- You may not further distribute the material or use it for any profit-making activity or commercial gain

- You may freely distribute the URL identifying the publication in the public portal 


\title{
Synchronization of oscillators with long range interaction: Phase transition and anomalous finite size effects
}

\author{
Máté Maródi, ${ }^{1, *}$ Francesco d'Ovidio, ${ }^{2, \dagger}$ and Tamás Vicsek ${ }^{1, \$}$ \\ ${ }^{1}$ Department of Biological Physics, Eötvös University, Pázmány Péter Sétány 1A, Budapest, 1117 Hungary \\ ${ }^{2}$ Chaos Group and Quantum Protein Center, Department of Physics, Building 309, Danish Technical University, \\ DK-2800 Lyngby, Denmark
}

(Received 21 December 2001; published 25 July 2002)

\begin{abstract}
Synchronization in a lattice of a finite population of phase oscillators with algebraically decaying, nonnormalized coupling is studied by numerical simulations. A critical level of decay is found, below which full locking takes place if the population contains a sufficiently large number of elements. For large number of oscillators and small coupling constant, numerical simulations and analytical arguments indicate that a phase transition separating synchronization from incoherence appears at a decay exponent value equal to the number of dimensions of the lattice. In contrast with earlier results on similar systems with normalized coupling, we have indications that for the decay exponent less than the dimensions of the lattice and for large populations, synchronization is possible even if the coupling is arbitarily weak. This finding suggests that in organisms interacting through slowly decaying signals such as light or sound, collective oscillations can always be established if the population is sufficiently large.
\end{abstract}

DOI: 10.1103/PhysRevE.66.011109

PACS number(s): 05.40.-a, 05.45.Xt

\section{INTRODUCTION}

Synchronization is one of the most fascinating nonlinear phenomena appearing in a wide range of fields. Examples cover physical systems (network of Josephson junctions [1]), oscillating chemical reactions [2], molecular turnover in allosteric enzymes [3], a variety of biological observations (synchronous flashing of fireflies [4], menstrual synchrony [5], metabolic activity in yeast cells [6,7], and other various physiological processes [8]), and social phenomena (rhythmic applause [9]).

Models for rhythmic synchronization use populations of nonlinearly coupled oscillators [10-12]. These models might be grouped according to the nature of coupling. For instance, for pulsatile interaction integrate-and-fire oscillators provide a useful tool of description [13] while in the case of continuous interaction a system of coupled limit cycle oscillators has proved to be a good model [2].

An important aspect of synchronizing systems is the spatial dependence of the coupling between two oscillators. Two limit cases have been deeply investigated: (i) mean field (with each pair of oscillators interacting with a given coupling strength, independent of their position, (ii) oscillators on a lattice with interactions only between nearest neighbors. However, realistic systems are quite different. In many of the biologically relevant systems the signals carrying information about the phase of an oscillator decay as a function of the distance slowly. For example, the intensity of the sound or light signals decays as a power law, in particular, in the three-dimensional case as the inverse of square of the distance from the source. In Ref. [14] results have been pre-

\footnotetext{
*Electronic address: marodi@complex.elte.hu

†Electronic address: dovidio@fysik.dtu.dk

\#Electronic address: vicsek@angel.elte.hu
}

sented concerning the existence of a critical exponent below which full synchronization can be achieved. These results considered a coupling term normalized by the sum of the spatial coefficients and the limit of the population size going to infinity.

However, in a real system typically the interaction between two oscillators depends only on the spatial distance and the phase differences and thus there is no normalization coefficient. This change, trivial at first sight, introduces a peculiar dependence of the dynamics on the number of oscillators, and thus places the study of finite populations into relevant context.

In this paper synchronization in populations of phase oscillators with a non-normalized coupling term is studied. Our investigations are especially focused on the dependence of full locking and the mean field on the number of oscillators for different value of the exponent $\alpha$ describing the spatial decay of the interactions. The main result of our work is the identification (through numerical simulations and a simple analytical argument) of critical values of $\alpha$ below which full synchronization, or in more general, collective oscillations can take place if the population is sufficiently large. We remark that very few works in our knowledge has approached the problem of finite size populations.

The paper is organized as follows. In Sec. II we summarize previous results on the versions of the model of our studies, the Kuramoto model. In Sec. III we explore the boundary of full locking in the plane of the decay exponent $\alpha$ and the number $N$ of oscillators, at different coupling values. We find that if $\alpha$ is below a critical value $\alpha_{c}(K)$ full synchronization can be achieved if the system is sufficiently large, i.e., above a critical size $N$. We argue, however, that this information is partial for real systems, since bulk oscillations can take place also if the system is not completely locked. We thus study in Sec. IV the behavior of the mean field. It appears then that a phenomenon close to a thermo- 
dynamic phase transition takes place looking at the mean field changing $\alpha$, with a discontinuity developing for $N$ going to infinity and increased fluctuations around the transition point. For $K$ going to zero, analytical arguments and numerical simulations indicate that the transition point occurs at $\alpha$ equal to the number of dimensions of the lattice. To show the robustness and generality of the result, we finally study a system of phase oscillators where the diversity is given by thermal noise. In such system full locking is not strictly defined, but on the contrary a mean-field approach can be carried out in the same way as for oscillators with fixed parameter distribution and the same kind of phase transition appears. The system with thermal noise is very similar to the Heisenberg model with ferromagnetic interaction. We shall suggest that a thermodynamic formalism may be applied to study analytically the phenomenon discussed numerically in this paper.

We remark that the present model has been proposed for real populations of oscillators (among others, specific examples are the above mentioned works on Josephson junctions [1] and synchronized applause [9]). In these cases, and when spatial dependences are included, our results have direct implications. They suggest that whenever the coupling signals of these systems decay slower than the number of spatial dimensions (like, for example, light or sound), collective oscillations always arise if the population is sufficiently large, however, weak the coupling is.

\section{THE KURAMOTO MODEL: PREVIOUS RESULTS}

The original form of the Kuramoto model was introduced to describe oscillating chemical reactions $[15,16]$. Later it proved to be useful in modeling a wide variety of processes (see, e.g., Ref. [17]). The basic concept is a population of $N$ interacting oscillators coupled via nonlinear phase-difference minimizing interaction. In general the equation of motion of the phase $\phi_{i}$ of the $i$ th oscillator is

$$
\dot{\phi}_{i}=\omega_{i}+\sum_{j} \Gamma_{i j}\left(\phi_{j}-\phi_{i}\right)
$$

where $\omega_{i}$ is the natural frequency of the $i$ th oscillator, $\Gamma(\phi)$ is the two-oscillator interaction and the sum goes over a suitably defined subset of the population depending on the actual model. The system can be further simplified choosing, without loss of generality, a frame of reference rotating at the average natural frequency $\bar{\omega}_{i}=\omega_{0}$ and rescaling the time and the coupling constant by the inverse of the standard deviation of the natural frequencies. The distribution of natural frequencies becomes then centered in zero and its standard deviation normalized.

Macroscopic states of this system can be characterized by a real order parameter:

$$
z(t)=\left|\frac{1}{N} \sum_{j=1}^{N} e^{\mathrm{i} \phi_{j}(t)}\right| .
$$

Another approach to synchronization is the investigation of frequency-locked states, i.e., when all $\dot{\phi}_{i}(t)$ approach asymptotically the same value.

Two basic setups of the Kuramoto model have been studied extensively so far. First, let us consider the case when

$$
\Gamma_{i j}(\phi)=\frac{K}{N} \sin (\phi)
$$

which defines a mean-field interaction, where $K>0$ is the coupling strength. Now the summation in Eq. (1) goes over the whole population. Let us assume that the distribution of the natural frequencies $\omega_{i}$ is symmetric about 0 , and is convex in that point. Under these conditions the existence of a critical coupling $K_{c}$ was proved, above which partial synchronization is possible [18-20]. With the above conditions the frequency of the locked subset is equal to the mean of the natural frequency distribution.

The other well explored case is when the interaction is inherently local, i.e., nearest neighbors are coupled. Then the model takes the form

$$
\dot{\phi}_{i}=\omega_{i}+K \sum_{j \in\langle i\rangle} \sin \left(\phi_{j}-\phi_{i}\right)
$$

where the symbol $\Sigma_{j \in\langle i\rangle}$ denotes summing over all the nearest neighbors of the $i$ th oscillator. Strogatz and Mirollo proved that the probability of a phase-locked solution tends to zero as the number of oscillators in the system goes to infinity [21]. Also, they studied the more general case when the mean interaction exerted on one oscillator is uniformly bounded and the distribution of natural frequencies "sufficiently broadbanded" (for details see Ref. [21]). For these conditions they showed that in the $N \rightarrow \infty$ limit the probability of a phase-locked state is zero.

An interesting situation arises when the interaction decays in space as a power function,

$$
\dot{\phi}_{i}=\omega_{i}+\frac{K}{\eta} \sum_{j \neq i} \frac{1}{r_{i j}^{\alpha}} \sin \left(\phi_{j}-\phi_{i}\right),
$$

where $r_{i j}$ is the distance between the $i$ th and $j$ th oscillator and $\eta$ is a normalization coefficient. It is clear that Eq. (5) is equivalent to the mean-field case when $\alpha=0$. Also, when $\alpha \rightarrow \infty$, the model becomes the same as in the nearest neighbors case. So, changing the exponent $\alpha$, one can have a continuous transition between the two extremes. Knowing the result of full synchronization in the global coupling case for high $K$, and only local synchronization in the nearest neighbors coupling for any $K$, one may expect a critical value of $\alpha$ below which full locking may be achieved. This was shown by Rogers and Wille, who studied Eq. (5) setting $\eta=2 \Sigma_{j=1}^{(N-1) / 2} 1 / j^{\alpha}$ in the limit of $N$ going to infinity [14]. They demonstrated that critical values of $\alpha$ exist depending on the coupling strength $K$. A similar result holds for sinecircle maps as well [22]. It was also recently shown numerically that an adequate normalization $\eta$ removes the depen- 
dency of the exponent $\alpha$ and system size $N$ on the fraction of oscillators synchronized in frequency in the case $\alpha<d$ [23].

However, in most real systems the coupling between two oscillators does not depend on global information on the population, but only on the phases and the distance of the two oscillators. It is thus realistic for these cases to set the normalization coefficient to 1 . As an important consequence, the behavior of the oscillators then strongly depends on the size of the population.

Throughout this paper we study models where $\eta=1$. Before describing any numerical results, we remark that an estimation of a critical point can be obtained looking at Eq. (5) and considering the coupling term. It is then natural to expect that the point $\alpha=d$, where $d$ is the number of dimensions of the lattice, has to play a special role. In fact if $\alpha \geqslant d$ the coupling term is bounded, for every $N$ :

$$
\left|\sum_{j \neq i}^{N} \frac{1}{r_{i j}^{\alpha}} \sin \left(\phi_{j}-\phi_{i}\right)\right| \leqslant \sum_{j \neq i}^{\infty} \frac{1}{r_{i j}^{\alpha}}<\infty .
$$

However, for $\alpha \leqslant d$, in the limit of large $N$ the value of the coupling term may entirely dominate the difference in natural frequencies $\omega_{i}$. More precisely, there are regions of the phase space where this term may diverge for $N$ going to infinity, in particular, in those regions where the difference between phases is large. Hence, we expect that for $\alpha \leqslant d$ synchronization is enhanced enlarging the size of the system, independently of the (positive) value of the coupling constant $K$. This property and the effects concerning the population size result from setting the coupling without normalization, and follow directly from the divergence of the upper bound in relation (6).

\section{FINITE POPULATION SIZE AND FULL SYNCHRONIZATION BOUNDARIES}

In this section we explore the effect of changing the number of oscillators in a one-dimensional system, measuring the boundary of the full locking region in the $(\alpha, N)$ plane, for different $K$ values. From the brief discussion in the preceding section we may expect that for sufficiently low values of $K$ synchronization can be achieved if $N$ is sufficiently large, only if $\alpha<1$. On the contrary, this should not be possible for $\alpha>1$.

In order to identify the exact value of $\alpha$ at which the transition occurs it is helpful to consider the fraction $p$ of the oscillators that are locked at the main frequency $\omega_{0}$. We thus count how many oscillators asymptotically satisfy the condition,

$$
\dot{\phi}_{i}=\omega_{0},
$$

and we divide this number by $N$.

The plane $(N, \alpha)$ has been scanned in either vertical or horizontal direction, numerically integrating population of oscillators at those parameter values. The transition points were identified as the boundary of the region of full synchronization, i.e., measuring the transient $p$ and finding the parameter values where $p$ is no longer equal to 1 . Refinements

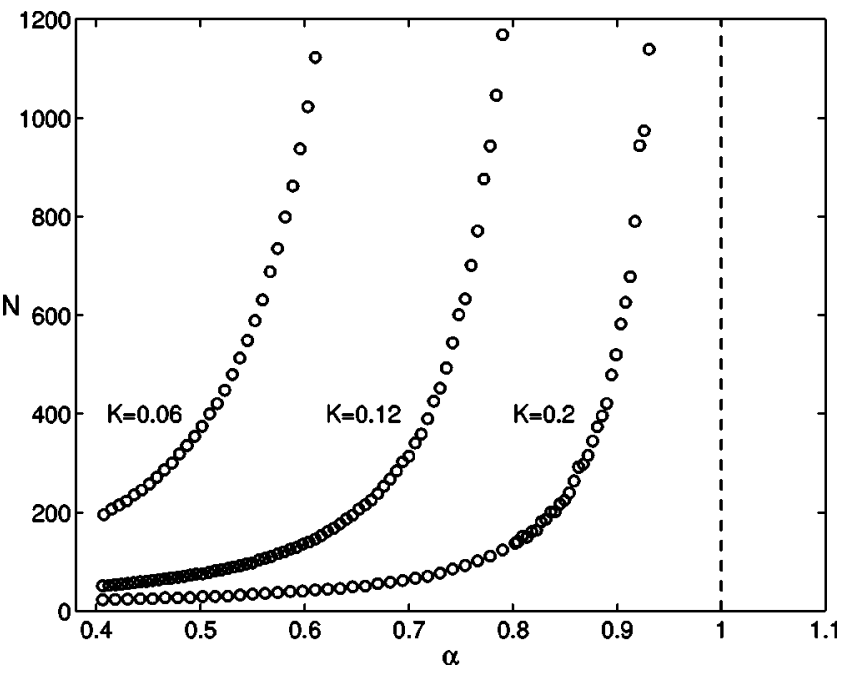

FIG. 1. Boundary of the full locking region in the $(N, \alpha)$ plane for different values of $K$. The line $\alpha=1$ is also shown.

up to $\Delta \alpha=0.01$ and $\Delta N=1$ were used. In all numerical computations in this paper the natural frequencies $\omega_{i}$ were chosen from a Gaussian distribution with expectation value $\bar{\omega}_{i}=0$ and unit variance. Since the value of $N_{c}$ can, in principle, depend on the position of the natural frequencies on the lattice, each point has been averaged on several configurations, especially for low values of $N$. We remark that, setting without loss of generality the main frequency $\omega_{0}$ to zero, the locked state appears as a steady state. To locate it, a direct integration method (Euler) has been used. This method is simple to implement and reliable, since Eq. (5) is clearly nonstiff, but requires a careful setting of the parameters for computing $p$ : in fact, the line $N_{c}$ corresponds to bifurcation points, where the transient time becomes more than exponentially long. Other methods, like continuation, could give a better estimation of the line but may be difficult to apply for

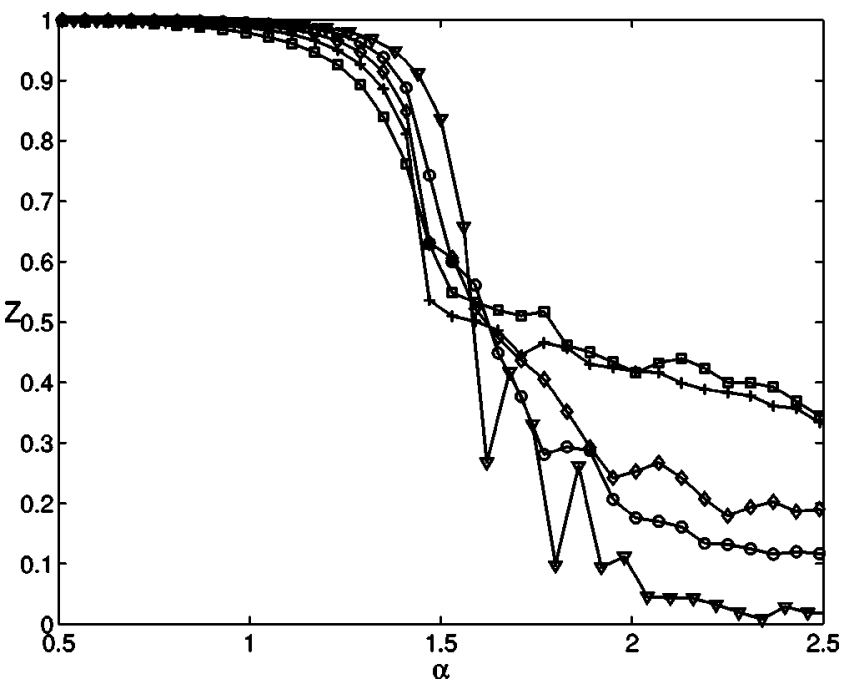

FIG. 2. Time average of the order parameter as a function of $\alpha$ in $d=1$. Simulation results for $N=50,100,200,500,1000$ (symbols are squares, pluses, diamonds, circles, and down triangles, respectively). The coupling is $K=1.0$. 


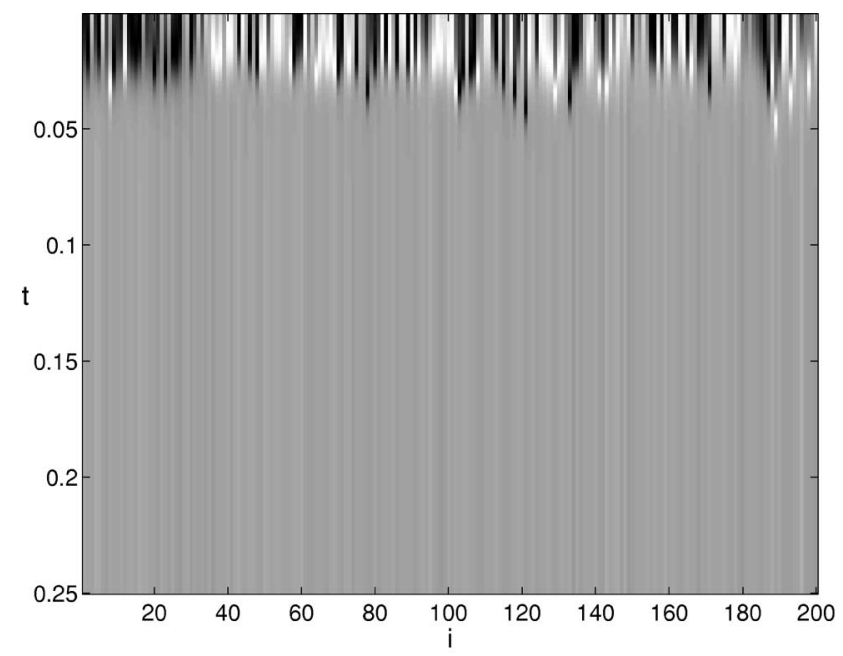

(a)

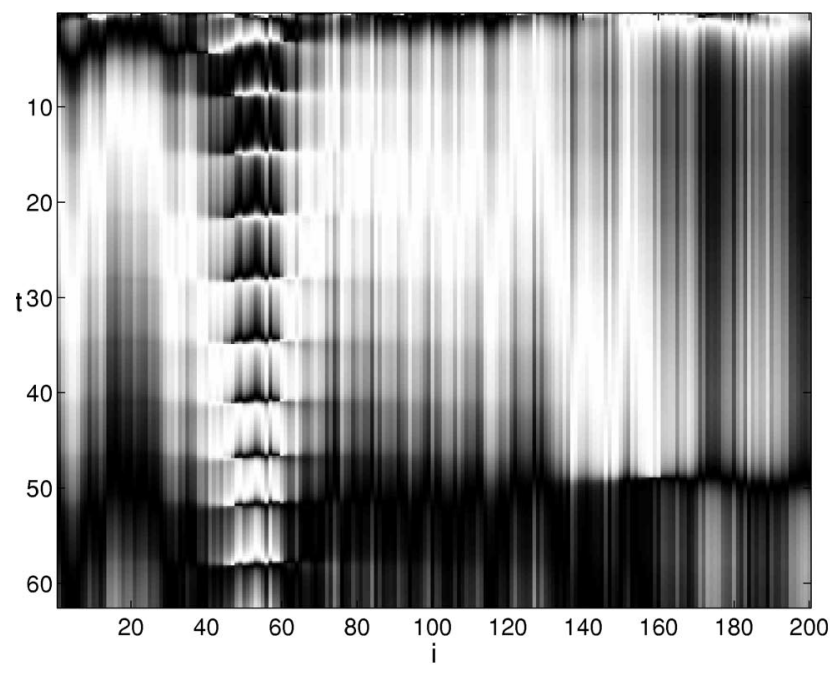

(c)

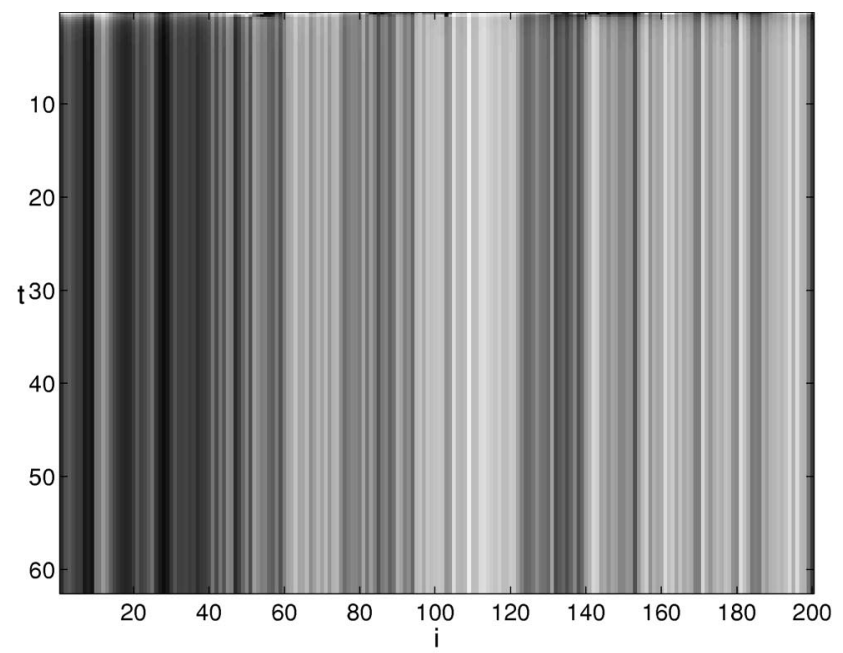

(b)

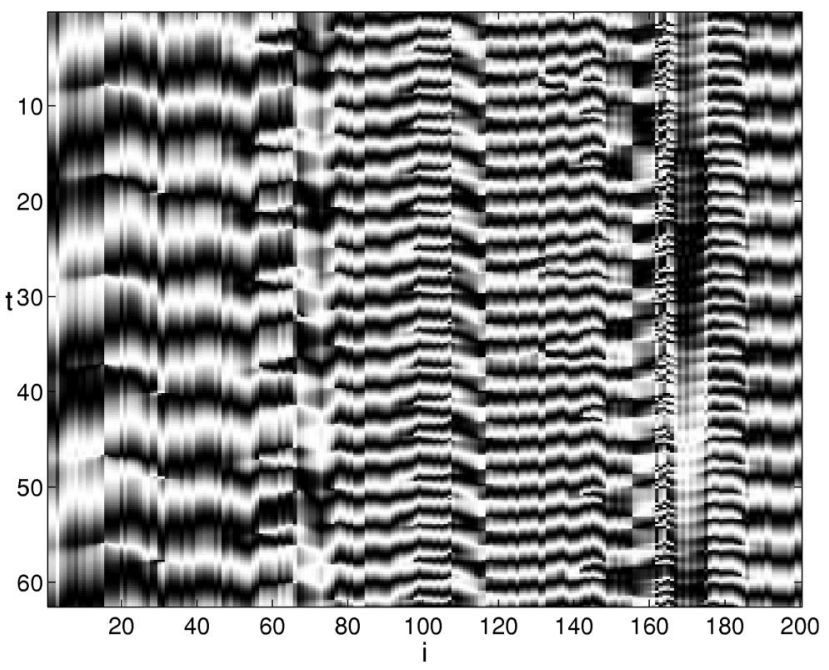

(d)

FIG. 3. Space-time plots representing the sine of the phase of the $i$ th oscillator for different $\alpha$ values: (a) $\alpha=0.5$, (b) $\alpha=1.5$, (c) $\alpha$ $=1.8$, and (d) $\alpha=2.5$. Gray scale indicates phases $\sin \left(\phi_{i}\right)$ from -1 (white) to 1 (black). Other parameters were set to $K=1.0$ and $N$ $=200$.

large systems, having to deal with matrices of the order of $N^{2 d}$.

For high coupling, full synchronization can appear at every $\alpha$ values. However, if $K$ is low we expect synchronization only for $\alpha$ values below the dimensions of the lattice. Results are plotted in Fig. 1. The region $\alpha>1$ is outside the boundary of full synchronization, confirming the discussion of Sec. I. The situation is more complex for $\alpha<1$. Here the effect of reducing the coupling can be indeed compensated by increasing the number of oscillators, up to a critical value of $\alpha$. Such critical value, however, seems to be in general different from 1 (the number of dimensions of the lattice), and depending on the coupling strength $K$. This is due to the fact that for small $K$ values, synchronization is still enhanced by a larger number of oscillators, although the system is not completely locked. The following section will investigate this situation, looking at the behavior of the mean field.

\section{COLLECTIVE OSCILLATIONS}

The results of the preceding section may be relevant for real systems. They suggest that in the case of oscillators coupled with a slowly decaying signal, full synchronization can be achieved as soon as the population is sufficiently large. However, the information that they give, describes only partially what may appear macroscopically. In this section we propose to approach the problem looking at the behavior of the mean field when $\alpha$ is changed. This criterion is less sharp than full synchronization: a transition cannot be seen for finite $N$ but, in analogy with thermodynamic phase transitions, looking at discontinuities for $N$ going to infinity. Nevertheless, as we shall see, the order parameter approach provides a robust and meaningful framework for describing the relations between synchronization and the decay of the coupling. Moreover, it allows to state a result in a simple way, connecting the critical value of the decaying of interac- 


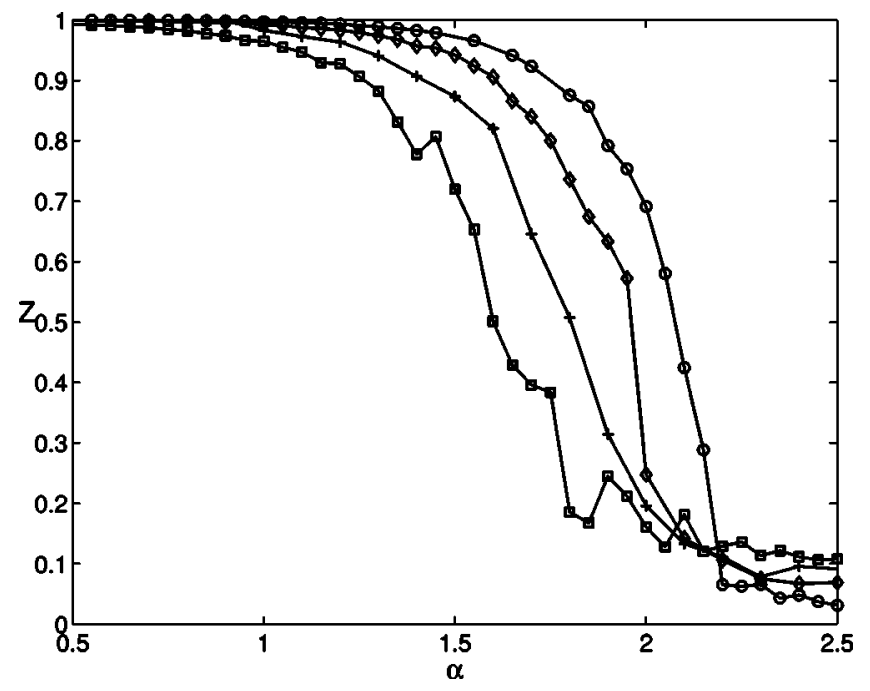

FIG. 4. Mean field $Z$ as a function of $\alpha$ for $d=2$ for $l \times l$ lattices. $l$ values are 7 (squares), 10 (pluses), 15 (diamonds), and 20 (circles). The coupling constant is $K=0.1$, and results were averaged over more configurations, especially for low $l$.

tion with the number of dimensions of the lattice.

Let us consider numerical simulations conducted on oneand two-dimensional lattices. We integrated the system of coupled differential Eq. (5) using the Euler method, time steps varied from 0.01 to 0.0005 relative time units (measured in comparison with the frequencies). The value of the order parameter is computed calculating at each time $z(t)$ $=\left|1 / N \sum_{j=1}^{N} e^{i \phi_{j}(t)}\right|$, discarding the first few thousand steps of integrations as transient. Averaging this value on a long time (typically, 20000 time steps) gives the measure of synchronization we use, $Z=\langle z(t)\rangle$.

One-dimensional results are plotted in Fig. 2, where one can observe the transition from highly synchronized states at low $\alpha$ values to unsynchronized states. The behavior of the system can be divided into three regimes. For $\alpha \lesssim 1$ the system is fully locked, while for $\alpha \gtrsim 2.5$ the order parameter $Z$ approaches a steady value. In between one finds the region of

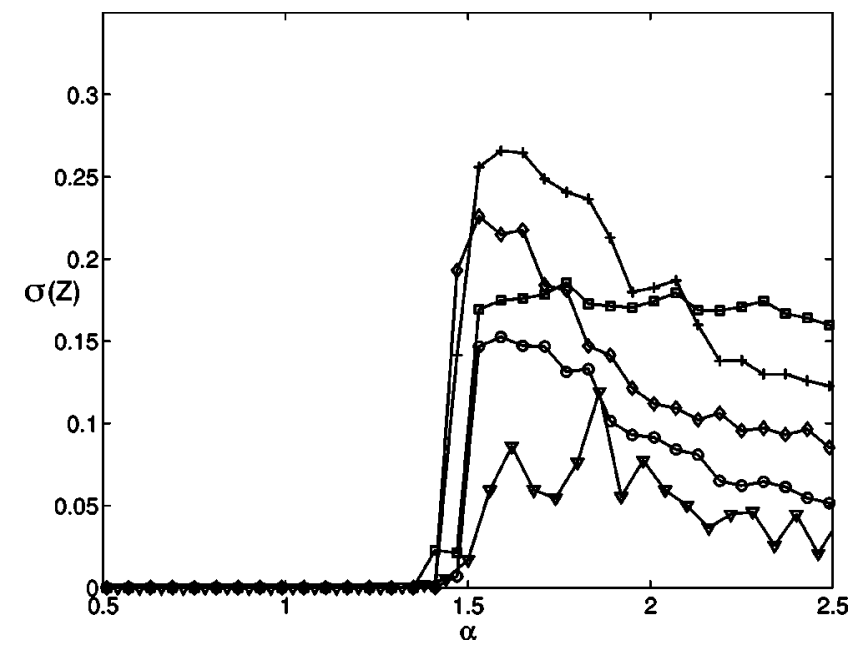

FIG. 5. The standard deviation $\sigma$ of the order parameter as a function of the $\alpha$ exponent for $K=1$. Symbols as in Fig. 2 .

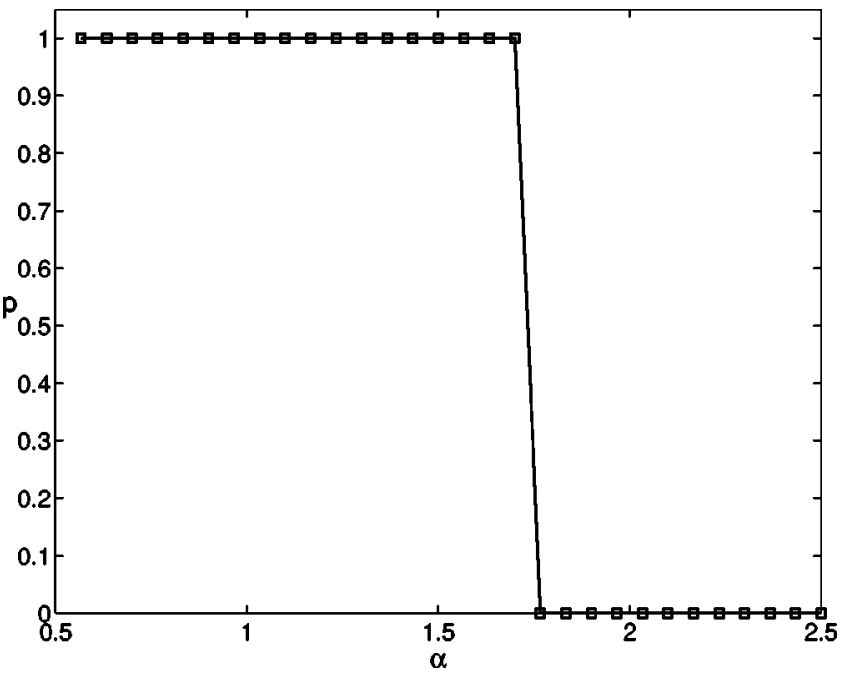

FIG. 6. The fraction $p$ of oscillators locked at the mean frequency for $K=1, N=200$.

transition. These three regions represent three different microscopic behaviors corresponding to the different macroscopic states (see Fig. 3). When $\alpha$ is low (e.g., $\alpha=0.5$ ) the system rapidly reaches the state of complete synchronization, when for all oscillators their frequencies become $\dot{\phi}_{i}=\omega_{0}$ $=0$. The next two subfigures represent the transitional region ( $\alpha=1.5,1.8)$. Here one can observe clusters arising first with increasing phase differences, then with synchronization holding only for finite times. This phenomenon leads to large fluctuations in the order parameter. Finally, in the unsynchronized region $(\alpha=2.5)$ there are only local synchronized groups, and clearly only very close oscillators influence each other.

The transition can be observed also in two dimensions (see Fig. 4). Simulations were conducted on a square lattice with the coupling constant set to $K=0.1$, otherwise all other parameters are the same as in $d=1$. As in the previous case, the transition becomes sharper as one increases $N$.

The phenomenon we present here is reminiscent of ther-

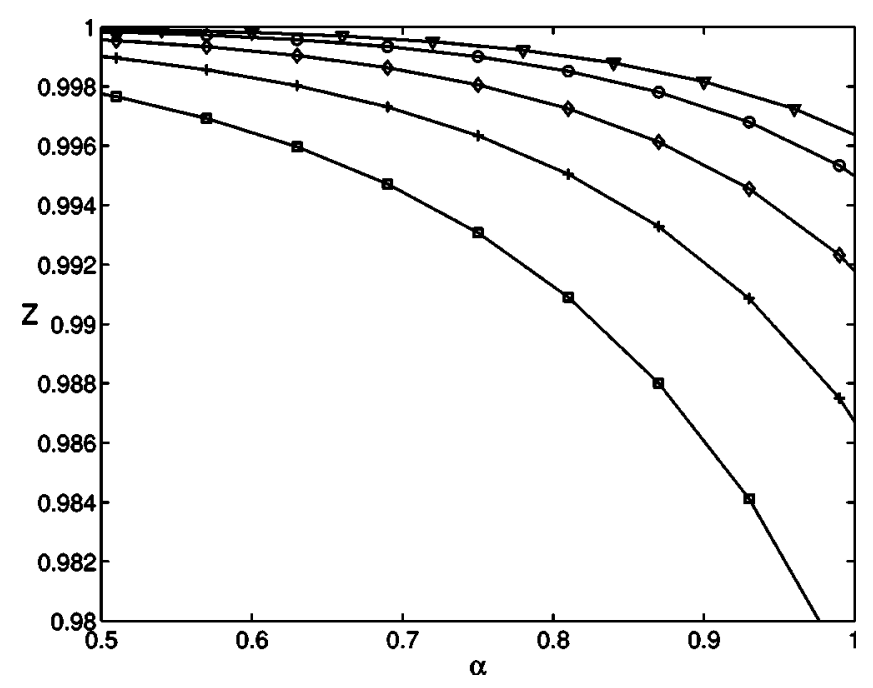

FIG. 7. Enlargement of Fig. 2. Synchronization region. 


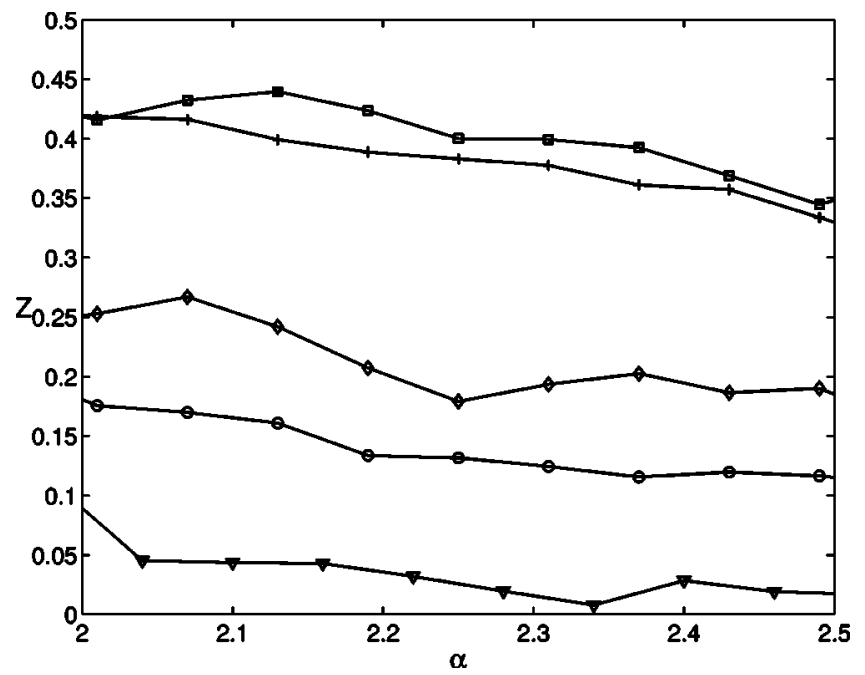

FIG. 8. Enlargement of Fig. 2. Incoherence region.

modynamic phase transitions in several features. First, the transition from unsynchronized state to a synchronized one breaks the original rotational symmetry of phases. Second, a phenomenon similar to the divergence of fluctuations close to the transition point can be observed also in this system, considering the standard deviation $\sigma$ of the order parameter time series (see Fig. 5).

It is interesting to compare the behavior of $Z$ (Fig. 2) with the plot obtained looking at the fraction of oscillators locked at the mean frequency (Fig. 6), used in the full locking approach. Although in the second case a discontinuity appears for finite $N$, one cannot distinguish between the transition region (with some unlocked oscillators but a strong mean field) and the region at high $\alpha$ (with developed incoherence and a low mean field).

Another effect that was missed looking at full locking only is the dependence of the mean field on $N$. From Fig. 7 one can see that at low $\alpha Z$ increases with $N$, since the

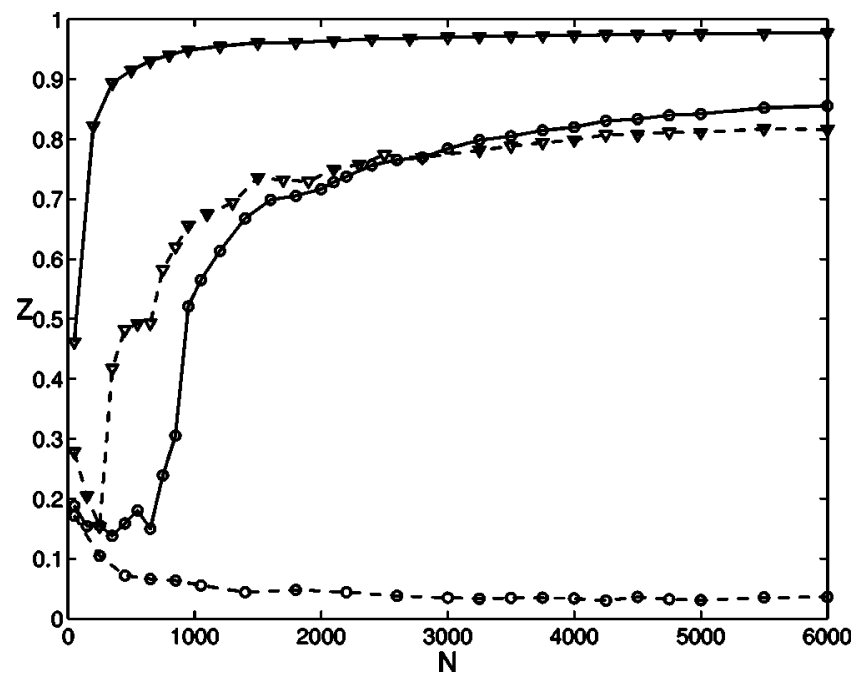

FIG. 9. Size dependence of order parameter for $\alpha$ below (continuous line, $\alpha=0.9$ ) and above (dashed line, $\alpha=1.1$ ) the critical value $\alpha=d=1$. The coupling is $K=0.2$ (triangles) and 0.1 (circles).

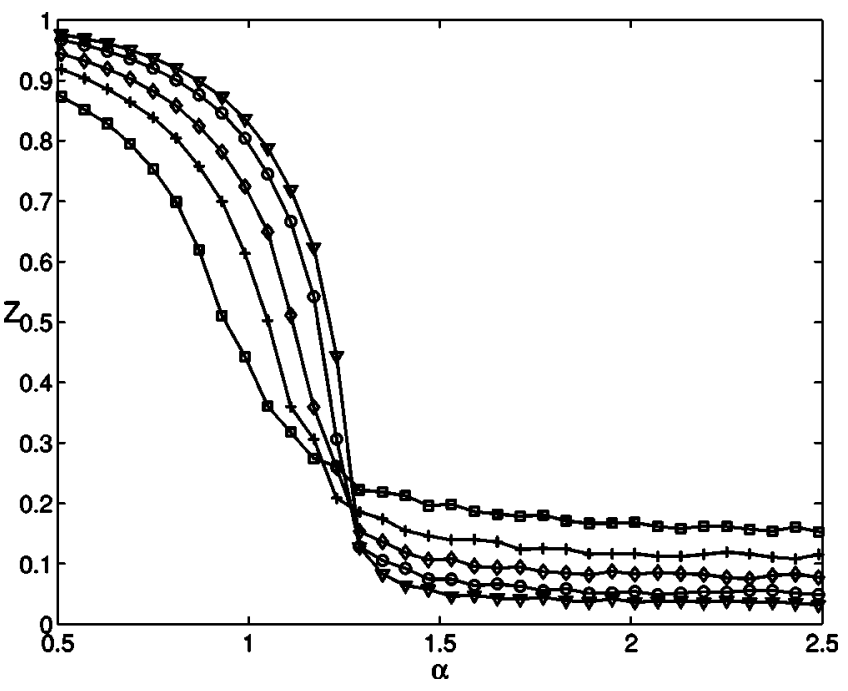

FIG. 10. Average of the order parameter as a function of $\alpha$ for identical oscillators with noise in $d=1$. Simulation results for $N$ $=50,100,200,500$, and 1000 (symbols are squares, pluses, diamonds, circles, and down triangles, respectively). The coupling is $K=1.0$.

oscillators rotate closer to each other (i.e, with smaller phase differences). We remark that this can happen even if the systems is not full locked. The effect on $Z$ is the opposite for incoherence (high $\alpha$ ). In this case, the decrease in the mean field is due to the fact that for high $N$, statistical fluctuations are reduced when averaging the uncorrelated phases over a larger number of the oscillators (Fig. 8).

The dependence of $Z$ over $N$ is also relevant when looking for a phase transition. In fact, it points to a discontinuity in the plot $Z=Z(\alpha)$ for $N$ going to infinity, as Figs. 2 and 4 suggest. In order to detect the phase transition, we perform simulations aimed at studying this effect. Results are plotted in Fig. 9. Considering as in Sec. II the convergence properties of the coupling term, and calling $d$ the number of dimensions of the lattice, they can be interpreted as follows.

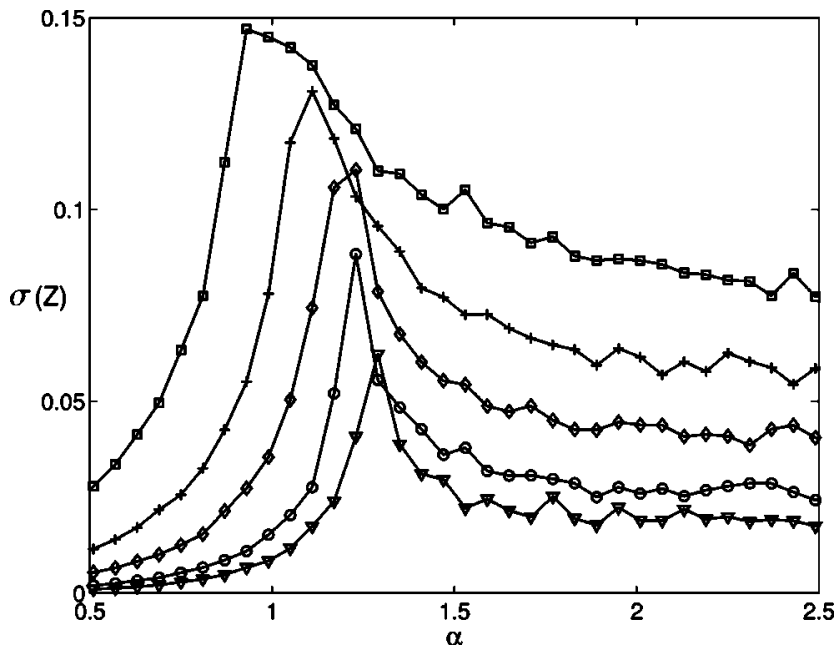

FIG. 11. Standard deviation $\sigma$ of the order parameter as a function of $\alpha$ for identical oscillators with noise in $d=1$. Symbols are the same as in Fig. 10. 


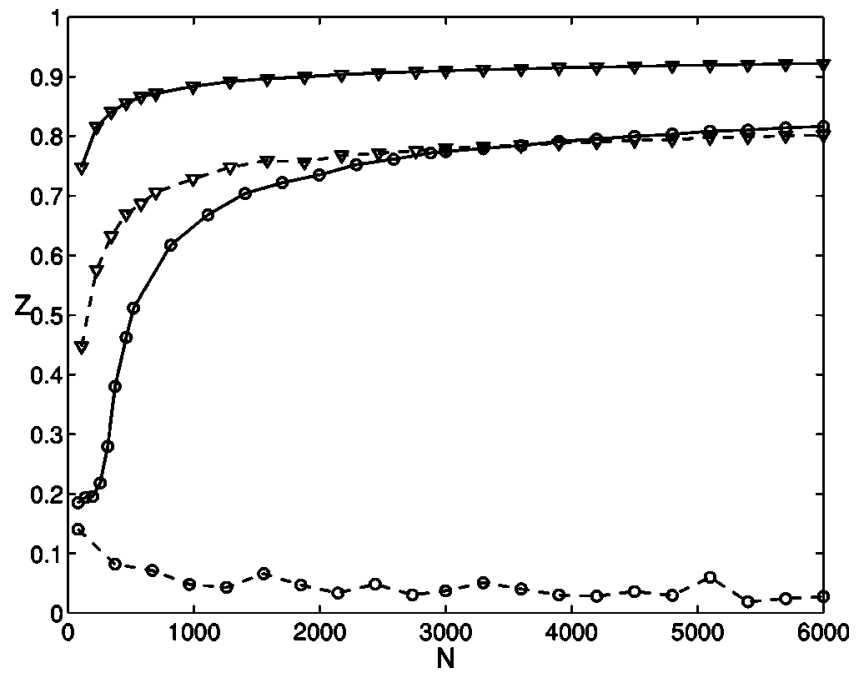

FIG. 12. Size dependence of order parameter for identical oscillators with noise for $\alpha$ below (continuous line) and above (dashed line) the critical value $\alpha=d=1$. The coupling is $K=1.0$ (triangles) and 0.5 (circles).

For $\alpha>d$ the coupling term is bounded for $N$ going to infinity. Hence, for $K$ sufficiently small, the system is incoherent and the mean field approaches 0 when $N$ increases. For $\alpha \leqslant 1$ the coupling term is unbounded for $N$ going to infinity (i.e., may diverge in some regions of the phase space). Hence, for any (fixed) $K$, however small, the mean field asymptotically approaches 1 for $N$ going to infinity. In mathematical terms

$$
\lim _{N \rightarrow \infty} \lim Z(\alpha)=\left\{\begin{array}{lll}
1 & \text { if } & \alpha \leqslant d, \\
0 & \text { if } & \alpha>d .
\end{array}\right.
$$

This double limit gives a compact and meaningful result on the synchronization properties of a system in relation to the decay of the coupling signal.

\section{POPULATION OF OSCILLATORS WITH THERMAL NOISE}

The analogy with thermodynamic phase transitions can actually be developed further. In this section we show that a similar transition takes place when the natural frequencies are equal, and randomness is introduced with thermal noise. The population of oscillators becomes then very close to a Heisenberg system with given temperature.

We rewrite the natural frequencies in the form

$$
\omega_{i}(t)=\omega_{0}+\xi_{i}(t)
$$

where $\xi_{i}(t)$ is the noise term chosen from some distribution. We choose $\xi_{i}(t)$ as a Gaussian distribution, such that $\overline{\xi_{i}(t)}$ $=0$ and $\overline{\xi_{i}(t) \xi_{j}\left(t^{\prime}\right)}=2 D \delta_{i j} \delta\left(t-t^{\prime}\right)$. It is clear that for every $t$ the natural frequencies $\omega_{i}$ have the same distribution as in the above discussed model. However, the realization of this distribution changes at each instant of time. The equations of motion for the oscillators thus become

$$
\dot{\phi}_{i}=\omega_{0}+K \sum_{j \neq i} \frac{1}{r_{i j}^{\alpha}} \sin \left(\phi_{j}-\phi_{i}\right)+\xi_{i}(t)
$$

Our simulations show that the phase transition from synchronized to unsynchronized state described in the preceding section takes place in this arrangement also (Figs. 10 and 11). Besides the transition, the size effects appear to remain valid in this case. For high $K$, synchronization does not depend on the relation between $\alpha$ and the lattice dimensions (Fig. 12, triangles). However, for low coupling there is indication that the system synchronizes only if $\alpha \leqslant d$ (Fig. 12, circles).

\section{DISCUSSION}

We have considered synchronization of phase oscillators on a lattice, looking at critical levels of spatial decay in the interaction. Especially, since the coupling term considered is not normalized, we studied the effect of changing the population size on synchronization. We examined the system by investigating full locking and the mean field. The two approaches have appeared to be complementary. The criterion of full locking allowed to precisely define the boundary of complete synchronization for finite population sizes. It gives, however, a very strong condition, requiring all oscillators to be exactly locked. For small coupling, this condition is not useful. If the coupling constant is small, synchronization can still be enhanced, below a critical $\alpha$ value, enlarging the size of the population: but some of the oscillators remain unlocked. We thus studied the system from a different point of view, that is, looking at the behavior of the mean field. In that case, a transition point is not strictly defined for finite population size. However, in the limit of infinite number of oscillators one can look, in analogy with thermodynamics, to the mean field as an order parameter and thus find critical values of the parameters where discontinuities appear. As one may expect, the value of the decay exponent equal to the number of lattice dimensions is then a good candidate for a transition point. At that value and below in fact, the coupling term is unbounded for an infinite size. That was supported by numerical simulations. As we pointed out, this gives a robust result for real systems: knowing only the number of lattice's dimensions and the decay in space of the coupling signal, one can predict if enlarging the size of the system eventually results in synchronization or not, even for arbitarily weak coupling constant.

We finally considered a system of oscillators in which the diversity is given not by fixed natural frequencies, but by noise. The notion of full locking is not useful for this system, but the mean-field approach can be carried out, and suggests the same features and critical point, at a decay exponent equal to the lattice's number of dimensions. Beside showing the robustness of the result, this last result is promising for 
an analytical treatment of the problem. In fact, the system with thermal noise is close to a Heisenberg spin system. We remark that an analytical investigation would be important. In fact, simulations are very time consuming, and allowed us to have indications of the phenomenon in a relatively small parameter region. Especially, the analysis of higher dimensional lattices, as well as lower coupling strength, would be interesting.

\section{ACKNOWLEDGMENTS}

F. d'Ovidio thanks E. Mosekilde and S. De Monte for the fruitful discussions. M. Maródi and T. Vicsek are grateful to A. Czirók and Z. Néda for many helpful discussions. Support from OTKA (Hungary, Grant No. T034995) is acknowledged. Some of the simulations were performed on the parallel cluster of MTA SZTAKI.
[1] P. Hadley, M.R. Beasley, and K. Wiesenfeld, Phys. Rev. B 38, 8712 (1988).

[2] Y. Kuramoto, Chemical Oscillations, Waves and Turbulence (Springer, Berlin, 1984).

[3] P. Stange, A.S. Mikhailov, and B. Hess, J. Phys. Chem. B 102, 6273 (1998).

[4] J. Buck, Q. Rev. Biol. 63, 265 (1988).

[5] M.K. McClintock, Nature (London) 392, 232 (1971).

[6] S. Dan $\phi$, P.G. Sørensen, and F. Hynne, Nature (London) 402, 320 (1999).

[7] S. Danф, F. Hynne, S.D. Monte, F. d'Ovidio, P.G. Sørensen, and H. Westerhoff, Faraday Discuss. 120, 261 (2002).

[8] L. Glass, Nature (London) 410, 277 (2001).

[9] Z. Néda, E. Ravasz, Y. Brechet, T. Vicsek, and A.-L. Barabási, Nature (London) 403, 849 (2000).

[10] A.T. Winfree, J. Theor. Biol. 16, 15 (1967).

[11] A. T. Winfree, The Geometry of Biological Time (Springer, New York, 1980).

[12] A. Pikovsky, M. Rosenblum, and J. Kurths, Synchronization
(Cambridge University Press, Cambridge, England, 2001).

[13] R.E. Mirollo and S.H. Strogatz, SIAM (Soc. Ind. Appl. Math.) J. Appl. Math. 50, 1645 (1990).

[14] J.L. Rogers and L.T. Wille, Phys. Rev. E 54, R2193 (1996).

[15] Y. Kuramoto, in International Symposium on Mathematical Problems in Theoretical Physics, Lecture Notes in Physics Vol. 39, edited by H. Araki (Springer, New York, 1975), pp. 420422.

[16] Y. Kuramoto, Physica A 106, 128 (1981).

[17] Z. Néda, E. Ravasz, T. Vicsek, Y. Brechet, and A.L. Barabási, Phys. Rev. E 61, 6987 (2000).

[18] Y. Kuramoto and I. Nishikawa, J. Stat. Phys. 49, 569 (1987).

[19] S.H. Strogatz, Physica D 143, 1 (2000).

[20] S.D. Monte and F. d'Ovidio, Europhys. Lett. 58, 21 (2002).

[21] S.H. Strogatz and R.E. Mirollo, Physica D 31, 143 (1988).

[22] S.E.de S. Pinto and R.L. Viana, Phys. Rev. E 61, 5154 (2000).

[23] M.S.O. Massunaga and M. Bahiana, e-print cond-mat/0201508. 